\title{
Stereoselective Synthesis of the Naturally Occurring 2-Pyranone Dodoneine
}

\author{
Paula Álvarez-Bercedo, ${ }^{[\mathrm{a}]}$ Eva Falomir, ${ }^{[\mathrm{a}]}$ Juan Murga, ${ }^{[\mathrm{a}]}$ Miguel Carda, ${ }^{[\mathrm{a}]}$ and J. Alberto \\ Marco*[b]
}

Keywords: Dodoneine / Oxygen heterocycles / 5,6-Dihydropyran-2-ones / Allylation / Asymmetric synthesis / Ring-closing metathesis

The first total synthesis of the naturally occurring dihydropyranone dodoneine is reported. Asymmetric allylations were used for the stereoselective generation of the two stereogenic centers. The pyranone ring was created by means of a ringclosing metathesis.

[a] Departamento de Química Inorgánica y Orgánica, Univ. Jaume I, E-12071 Castellón, Spain

Fax No.: +34-964-728214

E-mail: efalomir@qio.uji.es

[b] Departamento de Química Orgánica, Univ. de Valencia, c/D. Moliner, 50, E-46100 Burjassot, Spain.

Fax No.: +34-96-3544328.

E-mail: alberto.marco@uv.es

Supporting information for this article is available on the WWW under http://www.eurjoc.org/ or from the author.

\section{Introduction}

Dodoneine is a compound belonging to the ample group of naturally occurring 5,6-dihydropyran-2-ones, a compound class whose members exhibit many various biological activities. As a matter of fact, they have been shown to be cytotoxic, HIV protease inhibitors, apoptosis inductors, anti-leukemic agents, etc. Some of these pharmacological effects have been related to the Michael acceptor properties of the conjugated double bond. ${ }^{[1]}$ Dodoneine was very recently isolated from Tapinanthus dodoneifolius, a parasitic plant growing on a sheanut tree in Burkina Faso (West Africa), and was found to exhibit a vasorelaxant efect on preconstricted rat aortic rings. ${ }^{[2]}$ Its structure was assigned as 1 (Figure 1) on the basis of spectroscopic analyses combined with an X-ray diffraction analysis of a crystalline derivative. In the present communication, we report the first synthesis of this natural 2-pyranone.

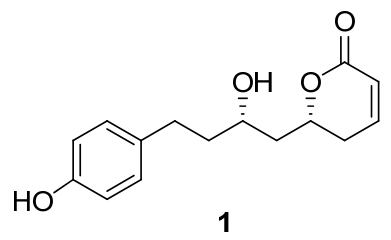

Figure 1

The retrosynthetic plan for pyranone 1, based on our previous experience with the syntheses of several members of this compound class, ${ }^{[1]}$ is shown in Scheme 1. Thus, 1 should be available via 2 from homoallyl alcohol $\mathbf{3}$ by means of a reaction sequence comprising acylation with acryloyl chloride, olefin metathesis and deprotection. ${ }^{[3]}$ Likewise, $\mathbf{3}$ was to be prepared from homoallyl alcohol 5 via aldehyde $\mathbf{4}$ by means of alcohol protection, oxidative cleavage of the olefinic bond and asymmetric allylation. The preparation of 5 from dihydro- $p$-coumaric acid $\mathbf{6}$ has already been reported. ${ }^{[4,5]}$ 


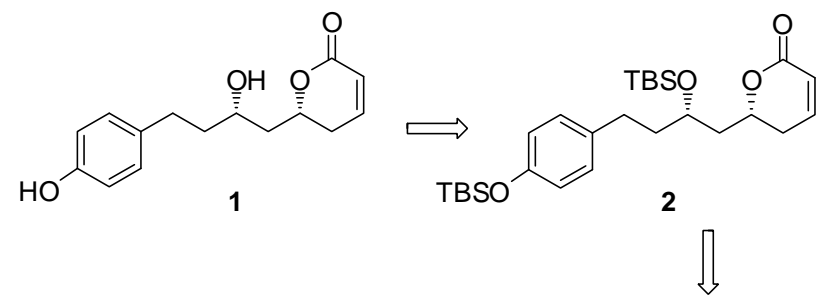<smiles>C=CCCCC(O)CCc1ccc(OCC(O)CCc2ccc(OC)cc2)cc1</smiles><smiles>C=CCC(O)CCc1ccc(OC(C)(C)C)cc1</smiles>

Scheme 1

\section{Results and Discussion}

Scheme 2 shows the specific details of the synthesis. Homoallyl alcohol 5 ( 95\% ee) was prepared from commercial dihydro-p-coumaric acid $\mathbf{6}$ according to the described procedures, ${ }^{[4,5]}$ which include an asymmetric Keck allylation ${ }^{[6]}$ of an intermediate silylated dihydro-p-coumaraldehyde. We also prepared homoallyl alcohol 5 by means of Brown's asymmetric allylboration ${ }^{[66,7]}$ of the same intermediate. However, we could not improve the reported results ${ }^{[5]}$ as the ee obtained was only $90 \%$ as determined by chiral HPLC ${ }^{[8]}$ Silylation of 5 to 7 (TBS $=$ tert-butyldimethylsilyl) and ozonolytic cleavage of the olefinic bond in the latter compound yielded aldehyde 4, which was then subjected in crude form to an asymmetric allylboration with the $(+)-\mathrm{Ipc}_{2} \mathrm{BCl} /$ allyl magnesium bromide reagent $\left(\mathrm{Ipc}=\right.$ diisopinocampheyl) ${ }^{[7]}$ This provided homoallyl alcohol 3, which was isolated as a single diastereomer (the minor stereoisomers went lost during the chromatographic separation). Sequential acylation of the latter with acryloyl chloride and ring-closing olefin metathesis of the resulting acrylate 8 using Grubbs first-generation catalyst $\mathbf{R u}-\mathbf{I}^{[9]}$ furnished pyranone 2. Cleavage of the two silyl groups in $\mathbf{2}$ was achieved by treatment with aqueous $\mathrm{HF}$ in $\mathrm{MeCN}$ to yield a compound with spectroscopic properties coincident with those reported for dodoneine $1 .^{[2]}$ 

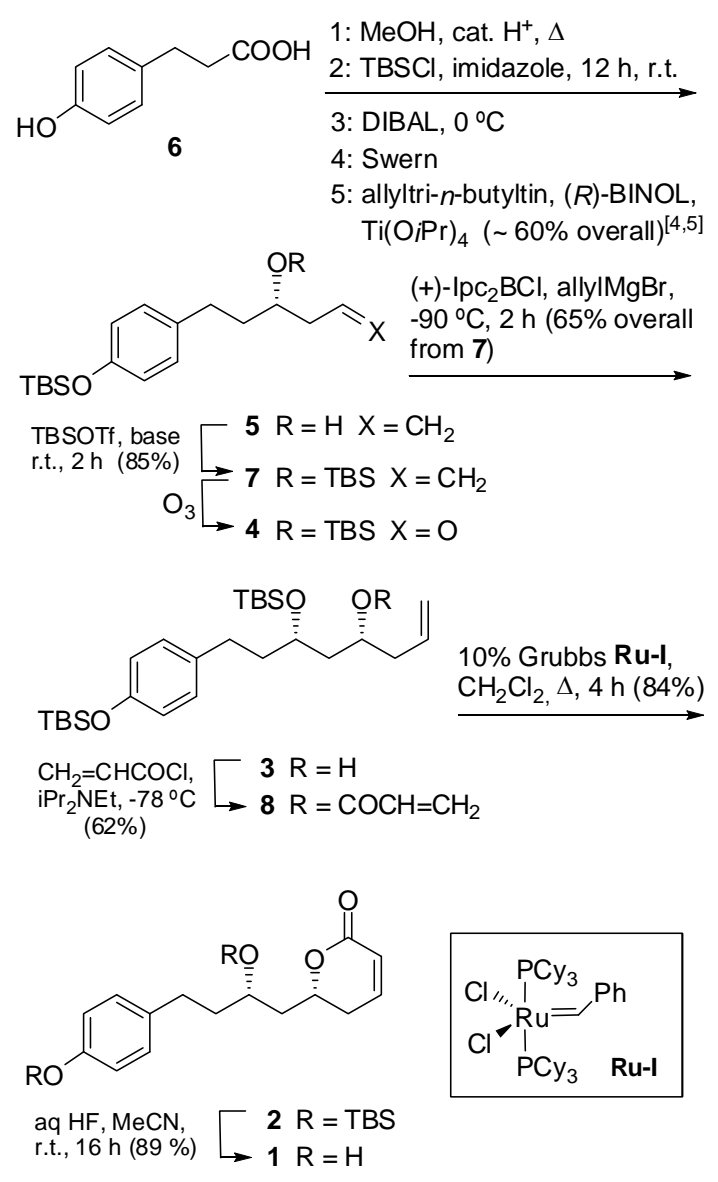

Scheme 2

\section{Experimental Section}

General experimental procedures: ${ }^{1} \mathrm{H} /{ }^{13} \mathrm{C}$ NMR spectra were measured at $500 / 125 \mathrm{MHz}$ in $\mathrm{CDCl}_{3}$ solution at $25{ }^{\circ} \mathrm{C}$. The signals of the deuterated solvent $\left(\mathrm{CDCl}_{3}\right)$ were taken as the reference (the singlet at $\delta 7.25$ for ${ }^{1} \mathrm{H}$ NMR and the triplet centered at $77.00 \mathrm{ppm}$ for ${ }^{13} \mathrm{C}$ NMR data). Carbon atom types $\left(\mathrm{C}, \mathrm{CH}, \mathrm{CH}_{2}, \mathrm{CH}_{3}\right)$ were determined with the DEPT pulse sequence. Mass spectra were run by the electron impact (EIMS, $70 \mathrm{eV}$ ) or the fast atom bombardment mode (FABMS, $m$ nitrobenzyl alcohol matrix) on a VG AutoSpec mass spectrometer. IR data are given only for compounds with significant functions $(\mathrm{OH}, \mathrm{C}=\mathrm{O})$ and were recorded as oily films on $\mathrm{NaCl}$ plates (oils) or as $\mathrm{KBr}$ pellets (solids). Optical rotations were measured at $25^{\circ} \mathrm{C}$. Reactions which required an inert atmosphere were carried out under $\mathrm{N}_{2}$ with flame-dried glassware. Et $\mathrm{O}_{2}$ and THF were freshly distilled from sodium/benzophenone ketyl and transferred via syringe. Dichloromethane was freshly distilled from $\mathrm{CaH}_{2}$. Tertiary amines were freshly distilled from $\mathrm{KOH}$. Toluene was freshly distilled from sodium wire. Commercially available reagents were used as received. Unless detailed otherwise, "work-up" means pouring the reaction mixture into brine, followed by extraction with the solvent indicated in parenthesis. If the reaction medium was acidic, an additional washing with $5 \%$ aq $\mathrm{NaHCO}_{3}$ was performed. If the reaction medium was basic, an additional washing with aq $\mathrm{NH}_{4} \mathrm{Cl}$ was performed. New washing with brine, drying over anhydrous $\mathrm{Na}_{2} \mathrm{SO}_{4}$ and elimination of the solvent under reduced pressure were followed by chromatography on a silica gel column $(60-200 \mu \mathrm{m})$ and elution with the indicated solvent mixture. Where solutions were filtered through a Celite pad, the pad was additionally washed with the same solvent used, and the washings incorporated to the main organic layer. Nonstandard acronyms are explained in the caption of Scheme 2.

1-(tert-Butyldimethylsilyloxy)-4-[(R)-3-(tert-butyl-dimethylsilyloxy)-hex-5-enyl]benzene (7): Alcohol 5 (613 mg, 2 mmol) was dissolved under $\mathrm{N}_{2}$ in dry $\mathrm{CH}_{2} \mathrm{Cl}_{2}(10 \mathrm{~mL})$ and treated sequentially with 2,6-lutidine $(350 \mu \mathrm{L}, 3 \mathrm{mmol})$ and TBSOTf $(575 \mu \mathrm{L}, 2.5 \mathrm{mmol})$. The reaction mixture was then stirred for $2 \mathrm{~h}$ at room temp. and worked up (extraction with $\mathrm{CH}_{2} \mathrm{Cl}_{2}$ ). Column chromatography on silica gel (hexanes-EtOAc, 19:1) afforded 7 (715 mg, 85\%): oil; $[\alpha]_{\mathrm{D}}=-2(c=1.4$, $\left.\mathrm{CHCl}_{3}\right) ;{ }^{1} \mathrm{H}$ NMR $\left(500 \mathrm{MHz}, \mathrm{CDCl}_{3}, 25{ }^{\circ} \mathrm{C}\right): \delta=7.05$ (apparent d, $\left.J=8.3 \mathrm{~Hz}, 2 \mathrm{H}\right), 6.78($ apparent d, $J=8.3 \mathrm{~Hz}, 2 \mathrm{H}), 5.85$ $(\mathrm{m}, 1 \mathrm{H}), 5.10-5.00(\mathrm{~m}, 2 \mathrm{H}), 3.77$ (quint, $J \sim 6 \mathrm{~Hz}, 1 \mathrm{H}), 2.70-2.60(\mathrm{~m}, 1 \mathrm{H}), 2.60-2.50(\mathrm{~m}, 1 \mathrm{H}), 2.30(\mathrm{~m}, 2 \mathrm{H}), 1.80-1.70(\mathrm{~m}$, 2H), $1.00(\mathrm{~s}, 9 \mathrm{H}), 0.93(\mathrm{~s}, 9 \mathrm{H}), 0.20(\mathrm{~s}, 6 \mathrm{H}), 0.07(\mathrm{~s}, 6 \mathrm{H}) ;{ }^{13} \mathrm{C} \mathrm{NMR}\left(125 \mathrm{MHz}, \mathrm{CDCl}_{3}, 25{ }^{\circ} \mathrm{C}\right): \delta=153.6,135.3,18.3,18.2$ $\left(\mathrm{C}_{\mathrm{q}}\right), 135.2,129.2(\times 2), 119.9(\times 2), 71.6(\mathrm{CH}), 116.8,41.9,38.8,31.0\left(\mathrm{CH}_{2}\right), 26.0(\times 3), 25.8(\times 3),-4.4(\times 4)\left(\mathrm{CH}_{3}\right) ; \mathrm{HR}$ EIMS m/z (\% rel. int.) $420.2880\left(\mathrm{M}^{+}, 1\right), 363\left(\mathrm{M}^{+}-t \mathrm{Bu}, 11\right), 221$ (100). Calcd. for $\mathrm{C}_{24} \mathrm{H}_{44} \mathrm{O}_{2} \mathrm{Si}_{2}, 420.2880$. 
(4R,6S)-6-(tert-Butyldimethylsilyloxy)-8-[4-(tert-butyldimethylsilyloxy)phenyl]-oct-1-en-4-ol (3). Olefin 7 (631 mg, 1.5 mmol) was dissolved in $\mathrm{CH}_{2} \mathrm{Cl}_{2}(25 \mathrm{~mL})$ and cooled to $-78^{\circ} \mathrm{C}$. A stream of ozone-containing air was then bubbled through the solution until complete consumption of the starting material (TLC monitoring). Ozone residues were then eliminated by bubbling a stream of $\mathrm{N}_{2}$, and the mixture was allowed to reach room temperature, treated with $\mathrm{PPh}_{3}(790 \mathrm{mg}, \sim 3 \mathrm{mmol})$ and allowed to stir for 2 hours. After solvent removal under reduced pressure, the crude residue was stirred for 10 min. under cold pentane $(10 \mathrm{~mL})$ and filtered. The solution was then concentrated under reduced pressure and the crude residue containing 4 was used directly in the next step.

Allylmagnesium bromide (commercial $1 \mathrm{M}$ solution in $\mathrm{Et}_{2} \mathrm{O}, 2 \mathrm{~mL}, 2 \mathrm{mmol}$ ) was added dropwise under $\mathrm{N}_{2}$ via syringe to a cooled solution of $(+)-\mathrm{Ipc}_{2} \mathrm{BCl}(800 \mathrm{mg}, \sim 2.5 \mathrm{mmol})$ in dry $\mathrm{Et}_{2} \mathrm{O}(12 \mathrm{~mL})$ (dry ice-acetone bath). After finishing the addition, the dry ice-acetone bath was replaced by an ice bath, and the mixture was stirred for $1 \mathrm{~h}$. The solution was allowed to stand, whereby precipitation of magnesium chloride took place. The supernatant solution was carefully transferred to another flask via canula. After cooling this flask at $-90{ }^{\circ} \mathrm{C}$, a solution of the crude aldehyde 4 from above in $\operatorname{dry~}^{\mathrm{Et}} \mathrm{t}_{2} \mathrm{O}(4 \mathrm{~mL})$ was added dropwise via syringe. The resulting solution was further stirred at $-90{ }^{\circ} \mathrm{C}$ for $2 \mathrm{~h}$. The reaction mixture was quenched through addition of phosphate $\mathrm{pH} 7$ buffer solution $(10 \mathrm{~mL}), \mathrm{MeOH}(10 \mathrm{~mL})$ and $30 \% \mathrm{H}_{2} \mathrm{O}_{2}(5 \mathrm{~mL})$. After stirring for 30 min., the mixture was poured onto satd. aq $\mathrm{NaHCO}_{3}$ and worked up (extraction with $\mathrm{Et}_{2} \mathrm{O}$ ). The residue was subjected to a careful column chromatography on silica gel (hexanes, then hexanes-EtOAc, 19:1 and 9:1) to afford pure $3(453 \mathrm{mg}, 65 \%$ overall from 7): oil; $[\alpha]_{\mathrm{D}}=+19.4\left(c=1.4, \mathrm{CHCl}_{3}\right) ;{ }^{1} \mathrm{H}$ NMR $\left(500 \mathrm{MHz}, \mathrm{CDCl}_{3}, 25^{\circ} \mathrm{C}\right): \delta=7.04$ (apparent $\mathrm{d}, J=8.2 \mathrm{~Hz}$, $2 \mathrm{H}), 6.77$ (apparent d, $J=8.2 \mathrm{~Hz}, 2 \mathrm{H}), 5.85(\mathrm{~m}, 1 \mathrm{H}), 5.15-5.10(\mathrm{~m}, 2 \mathrm{H}), 3.96(\mathrm{~m}, 1 \mathrm{H}), 3.83(\mathrm{~m}, 1 \mathrm{H}), 3.00$ (br s, $1 \mathrm{H}, \mathrm{OH})$, $2.65-2.55(\mathrm{~m}, 2 \mathrm{H}), 2.25(\mathrm{t}, J \sim 6.5 \mathrm{~Hz}, 2 \mathrm{H}), 1.90-1.60(\mathrm{br} \mathrm{m}, 4 \mathrm{H}), 1.00(\mathrm{~s}, 9 \mathrm{H}), 0.93(\mathrm{~s}, 9 \mathrm{H}), 0.20(\mathrm{~s}, 6 \mathrm{H}), 0.10(\mathrm{~s}, 6 \mathrm{H}) ;{ }^{13} \mathrm{C}$ $\operatorname{NMR}\left(125 \mathrm{MHz}, \mathrm{CDCl}_{3}, 25^{\circ} \mathrm{C}\right): \delta=153.7,134.7,18.2,18.0\left(\mathrm{C}_{\mathrm{q}}\right), 134.9,129.1(\times 2), 120.0(\times 2), 72.2,70.0(\mathrm{CH}), 117.6$, 42.4, 42.2, 39.8, $30.3\left(\mathrm{CH}_{2}\right), 25.9(\times 3), 25.7(\times 3),-4.1,-4.4(\times 2),-4.6\left(\mathrm{CH}_{3}\right) ; \mathrm{IR} v_{\max } 3450(\mathrm{br}, \mathrm{OH}) \mathrm{cm}^{-1}$; HR FAB MS $\mathrm{m} / \mathrm{z}$ 465.3236 $\left(\mathrm{M}+\mathrm{H}^{+}\right)$. Calcd. for $\mathrm{C}_{26} \mathrm{H}_{49} \mathrm{O}_{3} \mathrm{Si}_{2}, 465.3220$.

(4R,6S)-6-(tert-Butyldimethylsilyloxy)-8-[4-(tert-butyldimethylsilyloxy)phenyl]-oct-1-en-4-yl acrylate (8). Compound 3 $(325 \mathrm{mg}, 0.7 \mathrm{mmol})$ was dissolved under $\mathrm{N}_{2}$ in dry $\mathrm{CH}_{2} \mathrm{Cl}_{2}(20 \mathrm{~mL})$, cooled to $-78{ }^{\circ} \mathrm{C}$ and treated sequentially with N,Ndiisopropyl ethylamine $(1.4 \mathrm{~mL}, 8 \mathrm{mmol})$ and acryloyl chloride $(570 \mu \mathrm{L}, \sim 7 \mathrm{mmol})$. The reaction mixture was stirred at -78 ${ }^{\circ} \mathrm{C}$ until consumption of the starting material (TLC monitoring). Work-up (extraction with $\mathrm{CH}_{2} \mathrm{Cl}_{2}$ ) and column chromatography on silica gel (hexane-EtOAc, 19:1) provided $8(225 \mathrm{mg}, 62 \%)$ : oil; $[\alpha]_{\mathrm{D}}=-44.6\left(c=1.1, \mathrm{CHCl}_{3}\right) ;{ }^{1} \mathrm{H} \mathrm{NMR}$ $\left(500 \mathrm{MHz}, \mathrm{CDCl}_{3}, 25^{\circ} \mathrm{C}\right): \delta=7.02$ (apparent $\left.\mathrm{d}, J=8.2 \mathrm{~Hz}, 2 \mathrm{H}\right), 6.74$ (apparent d, $\left.J=8.2 \mathrm{~Hz}, 2 \mathrm{H}\right), 6.38(\mathrm{~d}, J=17.3 \mathrm{~Hz}, 1 \mathrm{H})$, $6.10(\mathrm{dd}, J=17.3,10.4 \mathrm{~Hz}, 1 \mathrm{H}), 5.80(\mathrm{~d}, J=10.4 \mathrm{~Hz}, 1 \mathrm{H}), 5.80-5.70(\mathrm{br} \mathrm{m}, 1 \mathrm{H}), 5.15-5.00(\mathrm{br} \mathrm{m}, 3 \mathrm{H}), 3.75(\mathrm{~m}, 1 \mathrm{H}), 2.65-$ 2.50 (br m, 2H), 2.45-2.30 (br m, 2H), 1.90-1.65 (br m, 4H), 0.98 (s, 9H), 0.91 (s, 9H), 0.18 (s, 6H), 0.07 (s, 3H), 0.05 (s, $3 \mathrm{H}) ;{ }^{13} \mathrm{C}$ NMR $\left(125 \mathrm{MHz}, \mathrm{CDCl}_{3}, 25{ }^{\circ} \mathrm{C}\right): \delta=165.6,153.6,134.9,18.2,18.1\left(\mathrm{C}_{\mathrm{q}}\right), 133.4,130.4,129.2(\times 2), 119.9(\times 2)$, 71.0, $68.9(\mathrm{CH}), 118.0,117.3,41.1,39.0,38.7,30.7\left(\mathrm{CH}_{2}\right), 25.9(\times 3), 25.8(\times 3),-4.4(\times 4)\left(\mathrm{CH}_{3}\right) ; \mathrm{IR} v_{\max } 1726(\mathrm{C}=\mathrm{O}) \mathrm{cm}^{-}$

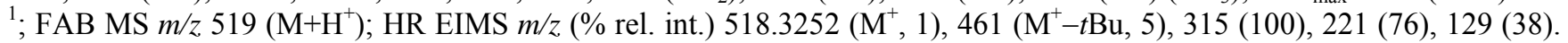
Calcd. for $\mathrm{C}_{29} \mathrm{H}_{50} \mathrm{O}_{4} \mathrm{Si}_{2}, 518.3247$.

(6R)-\{(S)-2-(tert-Butyldimethylsilyloxy)-4-[4-(tert-butyldimethylsilyloxy)phenyl]butyl\}-5,6-dihydropyran-2-one Diolefin 8 (130 mg, $0.25 \mathrm{mmol}$ ) was dissolved under $\mathrm{N}_{2}$ in dry, degassed $\mathrm{CH}_{2} \mathrm{Cl}_{2}(25 \mathrm{~mL})$ and treated with Grubbs catalyst $\mathrm{PhCH}=\mathrm{RuCl}_{2}\left(\mathrm{PCy}_{3}\right)_{2}(20 \mathrm{mg}$, ca. $0.025 \mathrm{mmol})$. The mixture was heated at reflux until consumption of the starting material (ca. $4 \mathrm{~h}$ ). Solvent removal under reduced pressure and column chromatography on silica gel (hexane-EtOAc, 19:1) yielded pyranone 2 (103 mg, 84\%): oil; $[\alpha]_{\mathrm{D}}=+38.2\left(c=2.3, \mathrm{CHCl}_{3}\right) ;{ }^{1} \mathrm{H}$ NMR $\left(500 \mathrm{MHz}, \mathrm{CDCl}_{3}, 25^{\circ} \mathrm{C}\right): \delta=7.04$ (apparent d, $J=$ $8.3 \mathrm{~Hz}, 2 \mathrm{H}), 6.88(\mathrm{~m}, 1 \mathrm{H}), 6.75$ (apparent d, $J=8.3 \mathrm{~Hz}, 2 \mathrm{H}), 6.03(\mathrm{br} \mathrm{d}, J=9.8 \mathrm{~Hz}, 1 \mathrm{H}), 4.60(\mathrm{~m}, 1 \mathrm{H}), 3.96$ (apparent quint, $J \sim 6 \mathrm{~Hz}, 1 \mathrm{H}), 2.65-2.55(\mathrm{~m}, 2 \mathrm{H}), 2.40-2.30(\mathrm{~m}, 2 \mathrm{H}), 2.15-2.05$ (m, 1H), 1.90-1.70 (br m, 3H), 0.99 (s, 9H), 0.91 (s, 9H), $0.18(\mathrm{~s}, 6 \mathrm{H}), 0.08(\mathrm{~s}, 3 \mathrm{H}), 0.05(\mathrm{~s}, 3 \mathrm{H}) ;{ }^{13} \mathrm{C}$ NMR $\left(125 \mathrm{MHz}, \mathrm{CDCl}_{3}, 25{ }^{\circ} \mathrm{C}\right): \delta=164.3,153.7,134.7,18.2,18.0\left(\mathrm{C}_{\mathrm{q}}\right), 144.8$, $129.1(\times 2), 121.5,120.0(\times 2), 75.2,68.2(\mathrm{CH}), 41.9,38.6,30.7,29.9\left(\mathrm{CH}_{2}\right), 25.9(\times 3), 25.7(\times 3),-4.5(\times 4)\left(\mathrm{CH}_{3}\right) ; \mathrm{IR} v_{\max }$ $1732(\mathrm{C}=\mathrm{O}) \mathrm{cm}^{-1}$; HR FAB MS m/z $491.3025\left(\mathrm{M}+\mathrm{H}^{+}\right)$. Calcd. for $\mathrm{C}_{27} \mathrm{H}_{47} \mathrm{O}_{4} \mathrm{Si}_{2}, 491.3013$.

(6R)-[(S)-2-Hydroxy-4-(4-hydroxyphenyl)butyl]-5,6-dihydropyran-2-one (1). A solution of 2 (49 $\mathrm{mg}, 0.1 \mathrm{mmol})$ in acetonitrile $(2.5 \mathrm{~mL})$ was treated at room temperature with aqueous $\mathrm{HF}(125 \mu \mathrm{L}, 48 \%$ in water, 30 equiv). The mixture was stirred for 16 hours. After removal of all volatiles under reduced pressure, column chromatography of the residue on silica gel (hexanes-EtOAc, 1:1, then EtOAc) afforded dodoneine $1(23 \mathrm{mg}, 89 \%)$ : amorphous solid; $[\alpha]_{\mathrm{D}}=+40.2(c=0.35$, $\left.\mathrm{CHCl}_{3}\right)$, lit. $^{2}[\alpha]_{\mathrm{D}}=+40.2\left(c=0.4, \mathrm{CHCl}_{3}\right) ;{ }^{1} \mathrm{H}$ NMR $\left(500 \mathrm{MHz}, \mathrm{CDCl}_{3}, 25^{\circ} \mathrm{C}\right): \delta=7.07$ (apparent d, $\left.J=8.6 \mathrm{~Hz}, 2 \mathrm{H}\right), 6.88$ $(\mathrm{dt}, J=9.7,4.5 \mathrm{~Hz}, 1 \mathrm{H}), 6.76$ (apparent d, $J=8.6 \mathrm{~Hz}, 2 \mathrm{H}), 6.02(\mathrm{dt}, J=9.8,2 \mathrm{~Hz}, 1 \mathrm{H}), 4.65(\mathrm{qd}, J=7.8,5.4 \mathrm{~Hz}, 1 \mathrm{H}), 3.89$ $(\mathrm{tt}, J \sim 7.8,4.4 \mathrm{~Hz}, 1 \mathrm{H}), 2.75-2.65(\mathrm{br} \mathrm{m}, 2 \mathrm{H}), 2.40-2.35(\mathrm{~m}, 2 \mathrm{H}), 2.02(\mathrm{dt}, J=14.5,8 \mathrm{~Hz}, 1 \mathrm{H}), 1.85-1.75(\mathrm{br} \mathrm{m}, 3 \mathrm{H}) ;{ }^{13} \mathrm{C}$ NMR $\left(125 \mathrm{MHz}, \mathrm{CDCl}_{3}, 25^{\circ} \mathrm{C}\right): \delta=164.2,154.1,133.7\left(\mathrm{C}_{\mathrm{q}}\right), 145.4,129.6(\times 2), 121.4,115.5(\times 2), 77.1,68.8(\mathrm{CH}), 42.2$, 39.5, 31.0, $29.7\left(\mathrm{CH}_{2}\right)$; IR $v_{\max } 3350(\mathrm{br}, \mathrm{OH}), 1698(\mathrm{C}=\mathrm{O}), 1515 \mathrm{~cm}^{-1}$; HR EIMS $\mathrm{m} / \mathrm{z}\left(\%\right.$ rel. int.) $262.1206\left(\mathrm{M}^{+}, 11\right), 244$ $\left(\mathrm{M}^{+}-\mathrm{H}_{2} \mathrm{O}, 6\right), 159(40), 107$ (100). Calcd. for $\mathrm{C}_{15} \mathrm{H}_{18} \mathrm{O}_{4}, 262.1205$.

Supporting Information (see footnote on the first page of this article): ${ }^{1} \mathrm{H}$ and ${ }^{13} \mathrm{C}$ NMR spectra of compounds 1, 2, 3, 7 and 8. 


\section{Acknowledgments}

Financial support has been granted by the BANCAJA-UJI foundation (projects P1-1A-2005-15 and P1-1B-2005-30) and by the Generalitat Valenciana (projects ACOMP07/023 and ACOMP07/025). P. A.-B. thanks the Universitat Jaume I for a research contract.

[1] a) For a recent review on the synthesis of naturally occurring representatives of this compound class, see: J. A. Marco, M. Carda, J. Murga, E. Falomir, Tetrahedron 2007, 63, 2929-2958. b) See also: V. Boucard, G. Broustal, J. M. Campagne, Eur. J. Org. Chem. 2007, 225-236.

[2] M. Ouedraogo, H. Carreyre, C. Vandebrouck, J. Bescond, G. Raymond, I. P. Guissou, C. Cognard, F. Becq, D. Potreau, A. Cousson, J. Marrot, J. M. Coustard, J. Nat. Prod. 2007, 70, 2006-2009.

[3] Ref. [1a] gives numerous examples of synthesis of naturally occurring 5,6-dihydropyran-2-ones by means of this methodology. For a more recent example, see: J. D. Umarye, T. Leßmann, A. B. García, V. Mamane, S. Sommer, H. Waldmann, Chem. Eur. J. 2007, 13, 3305-3319.

[4] a) The conversion of $\mathbf{6}$ into silylated dihydro-p-coumaraldehyde has been reported earlier albeit without experimental details: G. B. Jones, S. B Heaton, Tetrahedron: Asymmetry 1993, 4, 261-272. b) A. S. K. Hashmi, L. Schwarz, J. W. Bats, J. Prakt. Chem. 2000, 342, 40-51. c) S. Iimura, K. Manabe, S. Kobayashi, J. Org. Chem. 2003, 68, 8723-8725. d) D. Boschi, G. C. Tron, L. Lazzarato, K. Chegaev, C. Cena, A. Di Stilo, M. Giorgis, M. Bertinaria, R. Fruttero, A. Gasco, J. Med. Chem. 2006, 49, 2886-2897. e) A. B. Smith III, J. B. Sperry, Q. Han, J. Org. Chem. 2007, 72, 68916900

[5] Alcohol 5 has been obtained earlier in 95\% enantiomeric excess by means of Keck's asymmetric allylation of the silylated dihydro-pcoumaraldehyde: P. A. Evans, J. Cui, S. J. Gharpure, Org. Lett. 2003, 5, 3883-3885.

[6] For some reviews on asymmetric allylations of carbonyl compounds, see: a) R. O. Duthaler, A. Hafner, Angew. Chem. Int. Ed. 1997, 36, 43-45. b) P. V. Ramachandran, Aldrichimica Acta 2002, 35, 23-35. c) S. E. Denmark, J. Fu, Chem. Rev. 2003, 103, 2763-2793. d) D. G. Hall, Synlett 2007, 16441655 .

[7] P. V. Ramachandran, G.-M. Chen, H. C. Brown, Tetrahedron Lett. 1997, 38, 2417-2420.

[8] Data taken from the Ph.D. Thesis of P. A. B. (in preparation).

[9] a) A. Fürstner, Angew. Chem. Int. Ed. 2000, 39, 3012-3043. b) L. Jafarpour, S. P. Nolan, Adv. Organometal. Chem. 2000, 46, 181-222. c) T. M. Trnka, R. H. Grubbs, Acc. Chem. Res. 2001, 34, 18-29. d) J. A. Love in Handbook of Metathesis (Ed: R. H. Grubbs), Wiley-VCH, Weinheim, 2003, vol. 2, pp. 296-322. e) R. H. Grubbs, Tetrahedron 2004, 60, 7117-7140. f) For uses of RCM in the synthesis of 5,6-dihydropyran-2-ones, see ref. 1a.

The first total synthesis of the naturally occurring dihydropyranone dodoneine is reported. Asymmetric allylations were used for the stereoselective generation of the two stereogenic centers. The pyranone ring was created by means of a ring-closing metathesis.

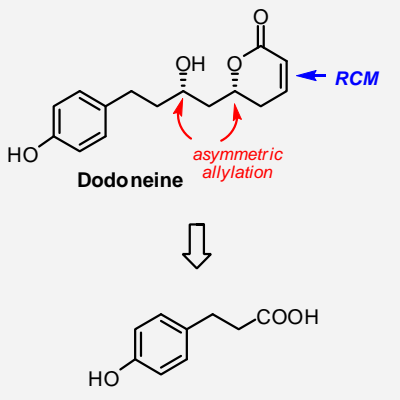

Dihydro- $p$-coumaric acid

((Key Topic))

Paula Álvarez-Bercedo, Eva Falomir,* Juan Murga, Miguel Carda, and J. Alberto Marco* ........ Page No. - Page No.

Stereoselective Synthesis of the Naturally Occurring 2-Pyranone Dodoneine

Keywords: 5,6-Dihydropyran-2-ones / Asymmetric allylation / Ring-closing metathesis 